\title{
Prospective study of stress, depression and personality in myasthenia gravis relapses
}

\author{
Anca Bogdan ${ }^{1}$, Carolina Barnett ${ }^{1,2}$, Abdulrahman Ali ${ }^{3}$, Mohammed AlQwaifly ${ }^{4}$, Alon Abraham5 ${ }^{5}$ Shabber Mannan ${ }^{1}$,
} Eduardo $\mathrm{Ng}^{1}$ and Vera Bril ${ }^{1,6^{*}}$ (D)

\begin{abstract}
Background: Psychopathology and personality traits may influence the course of autoimmune disorders. With this prospective longitudinal cohort study, we aimed to assess personality, stress and depression in myasthenia patients who relapse and those who remain stable or improve (non-relapsers).

Method: We collected data from 155 consecutive adult patients with confirmed MG attending the Neuromuscular Clinic, Toronto General Hospital, between March 2017 and July 2018, for this study. Patients were assessed at baseline and 6 months, or at the time of MG relapse. At both visits, the patients were assessed clinically and were asked to complete self-administered questionnaires for disease severity, chronic stress and depression. Personality type was assessed at baseline only. Relapsing patients were defined as those patients with MGIl score increasing by more than 5.5 points from visit 1 to visit 2 .

Results: Relapsers had higher baseline scores for depression $(p=0.01)$ and the change in disease severity correlated with the change in depression score $(r=0.2534, p=0.0015,95 \% \mathrm{Cl}: 0.0980 .3961)$. Higher levels of stress at baseline and neuroticism predicted higher relapse rates ( $p=0.01$ and $p<.0001$, respectively). In the linear regression model, with change of the MGII score as the dependent variable, change in depression scores $(p=0.0004)$ and age $(p=$ 0.03) predicted change in disease severity.
\end{abstract}

Conclusions: Since emotional factors and personality type may influence $M G$, attention to these factors might improve care in MG patients.

Keywords: Myasthenia gravis, Relapse, Trigger factors, Stress, Depression, Personality

\section{Background}

Acquired myasthenia gravis (MG) is a chronic autoimmune disorder caused by an antibody-mediated impairment of neuromuscular transmission resulting in fluctuating muscle weakness [1]. The prevalence of the disease is estimated to be 10 to 20 cases per 100,000 population [2].

\footnotetext{
* Correspondence: vera.bril@utoronto.ca

'Ellen and Martin Prosserman Centre for Neuromuscular Diseases, Division of Neurology, Department of Medicine, Toronto General Hospital, University Health Network, University of Toronto, 5EC, Room 309200 Elizabeth St, Toronto, ON M5G 2G4, Canada

${ }^{6}$ Institute for Research and Medical Consultations, Imam Abdulrahman Bin Faisal University, Dammam, Saudi Arabia

Full list of author information is available at the end of the article
}

In addition to infection and medication, which are common triggers of MG exacerbations, psychopathology, personality and coping mechanisms may influence the course of the disease [3]. Maladaptive traits such as neuroticism are associated with passive copings and cause high-stress levels [4]. Severe and prolonged mental stress and emotional arousal can affect immune function and may lead to onset or relapses of MG [5]. Also, MG is an unpredictable disease with a relapsing and remitting course necessitating the use of chronic medication with potential side-effects that impair quality of life [6-8] and may cause psychological stress and predispose to depression [9].

(C) The Author(s). 2020 Open Access This article is licensed under a Creative Commons Attribution 4.0 International License, which permits use, sharing, adaptation, distribution and reproduction in any medium or format, as long as you give appropriate credit to the original author(s) and the source, provide a link to the Creative Commons licence, and indicate if changes were made. The images or other third party material in this article are included in the article's Creative Commons licence, unless indicated otherwise in a credit line to the material. If material is not included in the article's Creative Commons licence and your intended use is not permitted by statutory regulation or exceeds the permitted use, you will need to obtain permission directly from the copyright holder. To view a copy of this licence, visit http://creativecommons.org/licenses/by/4.0/. The Creative Commons Public Domain Dedication waiver (http://creativecommons.org/publicdomain/zero/1.0/) applies to the data made available in this article, unless otherwise stated in a credit line to the data. 
Patients with MG likely experience chronic dysregulation of the hormonal stress axis and the immune system, aggravating the disease itself but also leading to secondary psychopathological abnormalities. In a recent cross-sectional study, $17.3 \%$ of MG patients had depression, [10] although a wide range of depression rates among MG patients has been described previously [11-17].

We aimed to assess personality, stress and depression factors in MG patients with relapses compared with those who did not relapse in a prospective longitudinal cohort study hypothesizing a relationship between personality type, chronic stress, and the likelihood of relapse.

\section{Methods}

\section{Participants}

We invited all consecutive adult patients with confirmed MG attending the Prosserman Family Neuromuscular Clinic, Toronto General Hospital, between March 2017 and July 2018, to participate in this study. Patients were eligible if they were over 18 years old and able to understand the study procedures. The diagnosis of MG was confirmed by a neuromuscular physician (VB, CB) based on the clinical presentation, abnormal single fiber electromyography studies, and positive antibody titres, if available.

\section{Ethics approval and consent to participate}

The University Health Network Research Ethics Board approved the study and all patients provided written informed consent.

\section{Measures}

Patients were assessed at baseline and 6 months, or earlier if there was a relapse of MG. At both visits, the patients were assessed clinically and were asked to complete the Myasthenia Gravis Impairment Index (MGII) for disease severity, [18-20] the short version of the Trier Inventory for Assessment of Chronic Stress (TICS) $[21,22]$ and Beck's Depression Inventory - Second Edition (BDI-II) [23]. Additionally, patients completed the Big 5 Personality Inventory (NEO-PI-Revised) [24] at baseline only. Information on background medical and mood disorders was also recorded.

Patients were categorized as non-relapsers (stable/better) or relapsers (relapse/worsening) using the MGII score values from baseline to completion visits. Relapsers were defined as those patients with MGII score increasing by more than 5.5 points from visit 1 to visit 2 [20].

\section{Statistical analysis}

Analyses were performed using R, version 3.5.0. Results are presented as counts $(\mathrm{N})$ and proportions (\%) or means $+/$ - standard deviations as appropriate. We compared clinical and demographic variables in relapsers and non-relapsers. We calculated and compared the rate of relapse for patients with and without higher levels of stress and depression. For continuous variables, the differences between groups were analyzed with t-tests and we used chi-square tests to compare proportions. We used Pearson correlation coefficients to evaluate the correlation of disease severity with changes in stress level and depression. We built a multivariable linear regression model using the change in MGII as the dependent variable to find the association between change in disease severity, level of stress, depression and personality type. We also incorporated relevant demographic and clinical factors (age, sex, thymoma, MG type, change in medications, MGII at baseline) in the models. We retained the best fitting model, and reported the estimates and standard error for each variable. $P$-values < 0.05 were considered statistically significant for all analyses.

\section{Results}

A total of 179 patients entered the study and completed the first visit, and 155 patients returned all questionnaires after the second visit. Reasons patients were not enrolled included those who: were acutely ill, declined research, lived at a distance and would not return, had language barriers, or were senile. Of those completing the study, $51.6 \%$ were women. Age ranged from 22 to 85 years and MG duration from 1 to 46 years with a mean duration of $11.3 \pm 9.1$ years. Mean MGII (disease severity) was 14.0 after the first visit and 15.3 after the second visit; $82.6 \%$ of patients had generalized disease, and the others had ocular disease; about one quarter were diagnosed with thymoma and $56.1 \%$ had a thymectomy; 111 of 155 patients had antibody tests and AChR antibodies were found in $57.5 \%$ and anti-MuSK antibodies in $5.4 \%$. The demographics, personality type, and prevalence of stress and depression in the cohort at baseline have been described previously [10]. At baseline, $17.3 \%$ had depression (BDI-II $\geq 17$ ) and $11.7 \%$ had higher levels of stress (TICS $\geq 60$ ).

Of 155 patients, $33(21.3 \%)$ were relapsers; of the remaining 122 patients (non-relapsers), 19 (12.3\%) were better and $103(66.5 \%)$ were stable. At baseline, 6\% of non-relapsers, and $9 \%$ of relapsers had a diagnosed mood disorder: depression, anxiety, panic attacks.

Patients who had a relapse also had worsening in the BDI-II scores, but no significant change on the TICS scale. The proportion of MG patients with symptoms of depression at the end of the study was $27.3 \%$ among relapsers and $12.5 \%$ for non-relapsers $(p=0.01)$. Overall, comparing patients with (BDI-II $\geq 17$ ) and without (BDIII $<17$ ) depression at baseline, we found significant 
differences with regards to their age at onset (39.2 vs 49.4 years, $p=0.003)$, age at the time of the study $(50.5$ vs 60.0 years, $p=0.002$ ) and associated levels of stressTICS scores $(61.2$ vs $29.6, p<.0001)$. They had similar disease duration (11.4 vs 10.6 years) and genders were almost equally distributed $(54.2 \%$ females and $45.8 \%$ males). There were no significant baseline differences in the proportion of relapsers and non-relapsers receiving immunosuppressant medications. Patients with a relapse had a slightly higher, but insignificant, mean daily dose of prednisone $(17.2 \pm 13$ vs $12.8 \pm 8, p=0.15)$. Those who relapsed had more severe disease as measured by the MGII at baseline. Demographics of our patient cohort and clinical findings are shown in Table 1.

Baseline stress was associated with relapse rate: in patients with baseline TICS < 30 ("rarely" had a stressful event), the relapse rate was $13.9 \%$, increasing to $22.6 \%$ with TICS between 30 and 59 ("sometimes" had a stressful event) and $31.6 \%$ with TICS score $\geq 60$ ("often" had a stressful event) ( $p$ value $=0.01$, chi-square test). For those without depression at baseline (BDI 0-10) the relapse rate was $17.6 \%$, with mild mood disturbance (BDI
11-16) $33.3 \%$ and with greater depression (BDI $\geq 17$ ) $24.1 \%$ ( $p$ value $=0.04$, chi-square test). The change in disease severity had a weak correlation with change in depression score $(\mathrm{r}=0.2534, p=0.0015,95 \% \mathrm{CI}: 0.098$ $0.3961)$ but no correlation with change in chronic stress score.

Table 2 shows disease severity, stress, depression and relapse rate across personality types at baseline. No extroverted patients were found in this cohort. Significantly higher stress levels were observed for neuroticism and openness, and the highest relapse rate was associated with neuroticism although the patient numbers are small in this cohort. There were no important differences with regards to presence of infections, admissions, change in medication or occurrence of personal problems across personality types, but comorbidities were more frequent in the agreeableness group than the openness group (58.3\% versus $11.1 \%, p=0.03$ ).

In the linear regression model using change of the MGII score as the dependent variable, the following variables were included: age, sex, change in TICS score, change BDI-II score, personality type, disease duration,

Table 1 Comparison between MG relapsers and non-relapsers

\begin{tabular}{|c|c|c|c|c|}
\hline Variable & $\begin{array}{l}\text { Total } \\
(n=155)\end{array}$ & Non-relapsers $(n=122)$ & $\begin{array}{l}\text { Relapsers } \\
(n=33)\end{array}$ & $p$ value \\
\hline Age (y) & $58.5 \pm 14.0$ & $58.5 \pm 14.5$ & $58.7 \pm 12.3$ & 0.9 \\
\hline Sex F & $80(51.6)$ & $64(52.5)$ & $16(48.5)$ & 0.7 \\
\hline Disease duration (y) & $10.7 \pm 8.8$ & $10.7 \pm 8.6$ & $10.8 \pm 9.6$ & 1.0 \\
\hline Age of onset (y) & $47.8 \pm 15.7$ & $47.8 \pm 16.2$ & $47.9 \pm 14.1$ & 1.0 \\
\hline Generalized & $128(82.6)$ & 97 (79.5) & $31(93.9)$ & 0.05 \\
\hline MGll Score & $1.4 \pm 8.4$ & $-1.6 \pm 5.3$ & $12.2 \pm 7.7$ & $<0.0001$ \\
\hline Thymoma & $42(27.1)$ & $33(27.0)$ & $9(27.3)$ & 1.0 \\
\hline Thymectomy & $87(56.1)$ & $67(54.9)$ & $20(60.0)$ & 0.6 \\
\hline AchRAb & $64(57.7)$ & $53(60.2)$ & $11(47.8)$ & 0.2 \\
\hline MuSK Ab & $6(5.4)$ & $4(4.5)$ & $2(8.7)$ & 0.5 \\
\hline TICS-S & $-5.8 \pm 16.8$ & $-6.8 \pm 16.4$ & $-1.8 \pm 17.9$ & 0.12 \\
\hline BDI-II & $-0.4 \pm 5.3$ & $-1.0 \pm 5.2$ & $1.7 \pm 5.5$ & 0.01 \\
\hline Potential triggers & $67(43.2)$ & $52(42.6)$ & $15(45.5)$ & 0.8 \\
\hline Personal problems & $26(16.7)$ & $17(13.9)$ & $9(27.3)$ & 0.07 \\
\hline Other illnesses & $48(31.0)$ & $35(28.7)$ & $13(39.4)$ & 0.2 \\
\hline \multicolumn{5}{|l|}{ Medications (baseline) } \\
\hline Prednisone & $97(63)$ & $75(61)$ & $22(67)$ & 0.59 \\
\hline Prednisone dose/day (mg) & $13.8 \pm 9.7$ & $12.7 \pm 8.3$ & $17.2 \pm 13.2$ & 0.15 \\
\hline Azathioprine & $56(41)$ & $48(40)$ & $8(24)$ & 0.10 \\
\hline Mycophenolate & $26(17)$ & $22(18)$ & $4(12)$ & 0.40 \\
\hline Reduction in Immunosuppressant (baseline to follow up) & $48(31)$ & $40(33)$ & $8(24)$ & 0.32 \\
\hline
\end{tabular}

Values are means \pm SD or $n(\%)$

Statistics are calculated using unpaired two-tailed T-test for numerical data and Fisher/Chi-Square test for categorical values

For MGII, TICS and BDI, values are change scores from the baseline visit

Potential triggers: infections, medication change, hospital admissions

$\mathrm{Cl}$ for difference in prednisone dose: $[-0.2,0.089 .1] \mathrm{mg}$ (crosses 0 ) 
Table 2 Personality, disease severity, stress, depression and relapse rates in 155 MG patients

\begin{tabular}{lllllllll}
\hline Personality & \% of total & MGII & $\Delta$ MGII & TICS & $\Delta$ TICS & BDI-II & $\Delta$ BDI-II & Relapse Rate (\%) \\
\hline Agreeableness & 33 & 14.3 & 2.7 & 37.2 & -4.6 & 9.9 & -0.9 & 23.5 \\
Conscientiousness & 30 & 12.5 & 0.8 & 37.3 & -8.6 & 0.21 & -0.1 & 21.7 \\
Neuroticism & 3 & 21.8 & 6 & 48.5 & -15 & 11.5 & -5.3 & 50 \\
Openness & 34 & 14.5 & 0.3 & 45.9 & -3.7 & 11.1 & 0.1 & 17 \\
p-value & & 0.6 & 0.32 & 0.03 & 0.31 & 0.08 & 0.22 & $<\mathbf{0 . 0 0 0 1}$ \\
\hline
\end{tabular}

Categorial variables compared by chi-squared test and continuous variables by ANOVA

$\%$ of total is \% of total cohort

acute infections, change in medical treatment, personal problems, comorbidities and baseline MGII score. The final model had $\mathrm{R}^{2}: 0.15$ and Likelihood Ratio $=25.11$, with $p=0.0006$. The only variables predicting change in disease severity were change in depression scores $(p=$ $0.0004)$ and age $(p=0.03)$. Model estimates are found in Table 3.

\section{Discussion}

In this prospective, longitudinal cohort study, we found a positive association between MG relapses and depression. Baseline stress levels also predicted relapses. When adjusting for confounders, change in depression and age were significantly associated with relapses, although baseline severity on MGII was not a predictor in this study. A one-point increase in MGII score was associated with a 0.5 point increase in BDI-II score.

Our study has shown a higher prevalence of depression at $17 \%$ compared to the Canadian and global estimates in the adult population of 5.4 and $4.4 \%[25,26]$. Our increased rate is similar to previous reports of more

Table 3 Linear regression model with change in MGII as the dependent variable

\begin{tabular}{lllll}
\hline & Coefficient & SE & $t$ & $\operatorname{Pr}(>\mathrm{t})$ \\
\hline Intercept & -4.542 & 3.473 & -1.31 & 0.193 \\
Age & 0.104 & 0.049 & 2.09 & $\mathbf{0 . 0 3 8}$ \\
Sex, Male & 1.251 & 1.386 & 0.90 & 0.368 \\
Disease Duration & 0.020 & 0.076 & 0.26 & 0.793 \\
Personality C & -1.750 & 1.723 & -1.02 & 0.311 \\
Personality N & 5.990 & 4.301 & 1.39 & 0.161 \\
Personality O & -3.148 & 1.697 & -1.86 & 0.065 \\
$\Delta$ TICS & 0.016 & 0.041 & 0.42 & 0.678 \\
$\Delta$ BDI-II & 0.450 & 0.126 & 3.57 & $<\mathbf{0 . 0 0 1}$ \\
Medical Y & 1.776 & 1.357 & 1.31 & 0.193 \\
Personal Y & 2.385 & 1.895 & 1.26 & 0.210 \\
Comorbidities $Y$ & -1.383 & 1.250 & -0.92 & 0.360 \\
Baseline MGII & -0.083 & 0.050 & -1.674 & 0.096 \\
\hline Only change in depras & & & &
\end{tabular}

Only change in depression and age predicted disease severity in this patient cohort

SE standard error

$\Delta=$ change prevalent depression and other psychiatric issues in MG patients $[13,16,17]$. Interestingly, our MG patients with depression were younger, with an earlier disease onset and they had higher scores of associated stress and disease severity, likely due to different methods of assessing depression in different studies or different study cohorts.

We found that the rate of relapse increased with increasing stress scores at baseline. However, this finding is confounded by the correlation between disease severity at baseline and depression. Because patients who relapsed had higher disease severity scores at baseline, we cannot assess if high depression scores are triggering the flare-ups in this cohort. Previously it has been reported that onset of MG is triggered by physical/emotional stress in up to $20 \%$ of patients [5] and this is comparable to the $14.8 \%$ of patients in our cohort who reported a stressful event at onset of MG. Many patients report exacerbation of symptoms after experiencing mental stress (60.6\%), [5] but in our cohort, the relapse rate of $31.6 \%$ in those having a frequent stressor was lower and this finding might be related to the different MG populations studied as our patients were generally less affected outpatients.

We found a positive correlation of depression and disease severity. Others have reported that disease severity and stressful life events were associated with depressive symptoms [4] and poor quality of life correlated with symptoms of depression [5-7]. Also, it has been reported that depression in MG is associated with early stage of disease, lack of response to treatment, and use of corticosteroids [12]. Most of our patients were on chronic steroid therapy perhaps accounting for the patients' depressive state. Alterations in corticosteroid and/or catecholamine level in response to stressors may play a key role in the development of MG exacerbation [27] and flares in other autoimmune disorders [28]. Only about $15 \%$ of our patients recognized a stressful event related to family, work or personal injuries associated with the onset of the disease, but this result may be limited by recall bias. Other retrospective studies report that up to $80 \%$ of patients experience uncommon emotional stress before disease onset although this seems high 
given our results. However, not only does stress cause disease, but the disease itself also causes significant stress in the patients, creating a vicious cycle [29]. Therefore, it is very difficult to establish causal pathways between stress and MG severity.

The rate of relapse was $21.3 \%$ in our patient cohort and the overall disease severity was low as shown by the MGII values. Other studies have reported relapse rates up to $34 \%$, [30] MG crisis in $20-30 \%$, [31, 32] or even lower [33]. It is likely that the brief duration of follow-up of 6 months prevented observation of a higher relapse rate in our study cohort. Longer studies may be needed to further study this question. In our study, stress level and relapse rate differed across personality types. Neuroticism was associated with the highest scores of stress, depression, disease severity and relapse rate in our study, similar to previous findings, [4] but since only a few patients had neuroticism, our findings must be considered with caution.

A surprising finding in our study was the high score of depression and stress associated with openness, but this group of mostly men had the lowest relapse rate. An explanation may be that they had better coping mechanisms using a more problem-focused method of handling stressful experiences, [34] and having more emotional inhibition [35]. Research findings show positive relationships between openness and active coping and positive reinterpretation [36]. We found that the lowest rates of disease severity and depression were associated with conscientiousness. Previously, it was reported that adaptive personality traits (e.g., high extraversion and conscientiousness) were less affected by daily stresses, although other studies differ [4, 37].

Some limitations of our study are inclusion of patients with established MG who are followed in our clinic (and so being unable to determine if depression and stress preceded the diagnosis or developed later), the short duration of follow-up, the absence of socioeconomic data, the lack of formal psychiatric evaluation, potential confounding of MG symptoms by those with affective disorders, inclusion of relatively stable outpatients with limited disease severity and uncertainty about the temporal association of depression and MG triggers or relapses.

The strengths of our study are the prospective, longitudinal study design, the large number of patients recruited, the use of a validated measure of disease severity recognized scales for assessing depression and stress, and a less severely affected MG outpatient cohort that avoids overestimation of depression.

In summary, we found that baseline stress levels and increasing depression were associated with higher relapse rates in MG but that personality type did not clearly influence relapse rate.

\section{Conclusion}

Since emotional factors and personality type may influence MG, attention to these factors might improve care in MG patients.

\section{Abbreviations \\ MG: Myasthenia gravis; MGII: Myasthenia Gravis Impairment Index; TICS: Trier Inventory for Assessment of Chronic Stress, short version; BDI- \\ II: Beck's Depression Inventory - Second Edition; NEO-PI-Revised: Big \\ 5 Personality Inventory; AChR: Acetylcholine receptor; Anti-MuSK: Anti muscle specific kinase; $\mathrm{Cl}$ : Confidence interval}

\section{Acknowledgements}

We would like to thank Dr. Joseph L. Flanders, PhD, OPQ, Assistant Professor, McGill University for advice on appropriate scales to use for this study.

\section{Authors' contributions}

$A B$ wrote the protocol and informed consent form and did the ethics submission, collected data and wrote the manuscript. CB helped with the concept for the study, data analyses and writing of the manuscript. VB conceived the study, provided oversight to the protocol and informed consent development, ethics submission, data collection and analyses and writing of the manuscript. AA1, MA, AA2, SM and EN helped collect data and EN assisted with the ethics submission. All authors have read and approved the manuscript.

\section{Funding}

the Toronto General/Toronto Western Foundation provided funds to run this research project and fund the fellows and staff who performed the research. The funding body did not play any role in the design of the study and collection, analysis, and interpretation of data and in writing the manuscript.

\section{Availability of data and materials}

Individual de-identified participant data and related documents such as study protocol and statistical analysis plan will be shared with qualified investigators for a period of 12 months after publication of this paper, pending review and approval of all detailed requests by the UHN Research Ethics Board. All such requests should be directed to the corresponding author.

\section{Ethics approval and consent to participate}

Ethics approval was obtained from the University Health Network Research Ethics Board. All subjects provided written informed consent.

\section{Consent for publication}

not applicable.

\section{Competing interests}

C. Barnett has received consultancy fees from Akcea, Alexion, Grifols and Takeda. She has received research funding from Grifols and Octapharma. None of these are related to this study. V. Bril has received consultancy fees from CSL, UCB, Argenx, Akcea, Alexion, Alnylam, Grifols And Takeda. She has received research funding from Octapharma, UCB, Argenx, Baxalta and Grifols. None of these are related to this study. A. Bogdan, A. Ali, M. AlQwaifly, A. Abraham, S. Mannan and E. Ng declare no conflicts.

\section{Author details}

${ }^{1}$ Ellen and Martin Prosserman Centre for Neuromuscular Diseases, Division of Neurology, Department of Medicine, Toronto General Hospital, University Health Network, University of Toronto, 5EC, Room 309200 Elizabeth St, Toronto, ON M5G 2G4, Canada. ${ }^{2}$ Institute of Health Policy, Management and Evaluation, Dalla Lana School of Public Health, University of Toronto, Toronto, Canada. ${ }^{3}$ National Neuroscience Institute, King Fahad Medical City, Riyadh, Saudi Arabia. ${ }^{4}$ Qassim University, Buraydah, Al-Qassim, Saudi Arabia.

${ }^{5}$ Neuromuscular Diseases Unit of the Department of Neurology, Tel Aviv Sourasky Medical Center, The Sackler Faculty of Medicine, Tel Aviv University, 
Tel Aviv, Israel. ${ }^{6}$ Institute for Research and Medical Consultations, Imam Abdulrahman Bin Faisal University, Dammam, Saudi Arabia.

Received: 9 December 2019 Accepted: 21 May 2020

Published online: 29 June 2020

\section{References}

1. Keesey JC. Clinical evaluation and management of myasthenia gravis. Muscle Nerve. 2004;29:484-505. https://doi.org/10.1002/mus.20030.

2. Breiner A, Widdifield J, Katzberg HD, et al. Epidemiology of myasthenia gravis in Ontario, Canada. Neuromuscul Disord. 2015;26:41-6. https://doi. org/10.1016/j.nmd.2015.10.009.

3. Doering S, Henze T, Schüssler G. Coping with myasthenia gravis and implications for psychotherapy. Arch Neurol. 1993;50:617-20. https:/doi.org/ 10.1001/archneur.1993.00540060055018.

4. Afshar $\mathrm{H}$, Roohafza HR, Keshteli AH, et al. The association of personality traits and coping styles according to stress level. J Res Med Sci. 2015;20: 353-8.

5. Blum S, Lee D, Gillis D, et al. Clinical features and impact of myasthenia gravis disease in Australian patients. J Clin Neurosci. 2015;22:1 164-9. https:// doi.org/10.1016/j.jocn.2015.01.022.

6. Hoffmann S, Ramm J, Grittner U, et al. Fatigue in myasthenia gravis: risk factors and impact on quality of life. Brain Behav. 2016;6:e00538. https://doi. org/10.1002/brb3.538.

7. Braz NFT, Rocha NP, Vieira ÉLM, et al. Muscle strength and psychiatric symptoms influence health-related quality of life in patients with myasthenia gravis. J Clin Neurosci. 2018;50:41-4. https://doi.org/10.1016/j. jocn.2018.01.011.

8. Jeong A, Min J-H, Kang YK, et al. Factors associated with quality of life of people with myasthenia gravis. PLoS One. 2018;13:e0206754. https://doi.org/ 10.1371/journal.pone.0206754.

9. Kulaksizoglu IB. Mood and anxiety disorders in patients with myasthenia gravis: aetiology, diagnosis and treatment. CNS Drugs. 2007;21:473-81. https://doi.org/10.2165/00023210-200721060-00004.

10. Bogdan A, Barnett C, Ali A, et al. Chronic stress, depression and personality type in patients with myasthenia gravis. Eur J Neurol. 2020;27:204-9. https:// doi.org/10.1111/ene.14057.

11. Fisher J, Parkinson K, Kothari MJ. Self-reported depressive symptoms in myasthenia gravis. J Clin Neuromuscul Dis. 2003:4:105-8.

12. Suzuki $Y$, Utsugisawa $K$, Suzuki $S$, et al. Factors associated with depressive state in patients with myasthenia gravis: a multicentre cross-sectional study. BMJ Open. 2011;1:e000313. https://doi.org/10.1136/bmjopen-2011-000313.

13. Magni G, Micaglio GF, Lalli R, et al. Psychiatric disturbances associated with myasthenia gravis. Acta Psychiatr Scand. 1988;77:443-5.

14. Aysal F, Karamustafalioğlu O, Özçelik B, et al. The relationship of symptoms of anxiety and depression with disease severity and treatment modality in myasthenia gravis: a cross-sectional study. Noro Psikiyatr Ars. 2013;50:295300. https://doi.org/10.4274/npa.y5611.

15. Paul RH, Cohen RA, Goldstein JM, Gilchrist JM. Severity of mood, selfevaluative, and vegetative symptoms of depression in myasthenia gravis. J Neuropsychiatry Clin Neurosci. 2000;12:499-501. https://doi.org/10.1176/jnp. 12.4.499.

16. Sitek EJ, Bilińska MM, Wieczorek D, Nyka WM. Neuropsychological assessment in myasthenia gravis. Neurol Sci. 2009;30:9-14. https://doi.org/ 10.1007/s10072-008-0001-y.

17. Ybarra Ml, Kummer A, Frota ERC, et al. Psychiatric disorders in myasthenia gravis. Arq Neuropsiquiatr. 2011;69:176-9.

18. Barnett C, Bril V, Kapral M, et al. A conceptual framework for evaluating impairments in myasthenia gravis. PLoS One. 2014;9:e98089. https://doi.org/ 10.1371/journal.pone.0098089.

19. Barnett C, Bril V, Kapral M, et al. Development and validation of the myasthenia gravis impairment index. Neurology. 2016;87:879-86. https://doi. org/10.1212/WNL.00000000000002971.

20. Barnett C, Bril V, Kapral M, et al. Myasthenia gravis impairment index responsiveness, meaningful change, and relative efficiency. Neurology. 2017; 89:2357-64. https://doi.org/10.1212/WNL.0000000000004676.

21. Allen AP, Kennedy PJ, Dockray S, et al. The Trier social stress test: principles and practice. Neurobiol Stress. 2017;6:113-26. https://doi.org/10.1016/j.ynstr. 2016.11.001
22. Kirschbaum C, Pirke KM, Hellhammer DH. The "Trier social stress test"--a tool for investigating psychobiological stress responses in a laboratory setting. Neuropsychobiology. 1993;28:76-81. https://doi.org/10.1159/000119004.

23. Beck AT, Steer RA, Brown GK. Manual for the Beck Depression Inventory-II. 1996. San Antonio, TX: Psychological Corporation.

24. Costa PT. Jr, McCrae RR. The Revised NEO Personality Inventory (NEO-PI-R) 2008. In G. J. Boyle, G. Matthews, \& D. H. Saklofske (Eds.), The SAGE handbook of personality theory and assessment, Vol. 2. Personality measurement and testing (p. 179-198). Sage Publications, Inc. https://doi. org/10.4135/9781849200479.n9.

25. Pearson FS, Cleland CM, Chaple M, et al. Substance use, mental health problems, and behavior at risk for HIV: evidence from CJDATS. J Psychoactive Drugs. 2008;40:459-69. https://doi.org/10.1080/02791072.2008.10400652.

26. World Health Organization Depression and Other Common Mental Disorders. Global Health estimates. In: httpsapps.who.intirisbitstreamhandleWHO-MSDMER--eng.pdf;sequence. Accessed 18 Jul 2019.

27. Beydoun SR, Wang J, Levine RL, Farvid A. Emotional stress as a trigger of myasthenic crisis and concomitant takotsubo cardiomyopathy: a case report. J Med Case Rep. 2010;4:393. https://doi.org/10.1186/1752-1947-4-393.

28. Stojanovich L. Stress and autoimmunity. Autoimmun Rev. 2010;9:A271-6. https://doi.org/10.1016/j.autrev.2009.11.014.

29. Stojanovich L, Marisavljevich D. Stress as a trigger of autoimmune disease. Autoimmun Rev. 2008;7:209-13. https://doi.org/10.1016/j.autrev.2007.11.007.

30. Wang L, Zhang Y, He M. Clinical predictors for the prognosis of myasthenia gravis. BMC Neurol. 2017;17:77. https://doi.org/10.1186/s12883-017-0857-7.

31. Juel VC. Myasthenia gravis: management of myasthenic crisis and perioperative care. Semin Neurol. 2004;24:75-81. https://doi.org/10.1055/s2004-829595.

32. Lacomis D. Myasthenic crisis. Neurocrit Care. 2005;3:189-94. https://doi.org/ 10.1385/NCC:3:3:189

33. Ramos-Fransi A, Rojas-Garcia R, Segovia S, et al. Myasthenia gravis: descriptive analysis of life-threatening events in a recent nationwide registry. Eur J Neurol. 2015;22:1056-61. https://doi.org/10.1111/ene.12703.

34. Kelly MM, Tyrka AR, Price LH, Carpenter LL. Sex differences in the use of coping strategies: predictors of anxiety and depressive symptoms. Depress Anxiety. 2008;25:839-46. https://doi.org/10.1002/da.20341.

35. Matud MP, Bethencourt JM, Ibánez I. Gender differences in psychological distress in Spain. Int J Soc Psychiatry. 2015;61:560-8. https://doi.org/10.1177/ 0020764014564801.

36. Penley JA, Tomaka J, Wiebe JS. The association of coping to physical and psychological health outcomes: a meta-analytic review. J Behav Med. 2002; 25:551-603.

37. Ayache D, da Costa IP. Personality traits and associated changes in women with lupus. Rev Bras Rheumatol. 2009:49:643-57.

\section{Publisher's Note}

Springer Nature remains neutral with regard to jurisdictional claims in published maps and institutional affiliations.
Ready to submit your research? Choose BMC and benefit from:

- fast, convenient online submission

- thorough peer review by experienced researchers in your field

- rapid publication on acceptance

- support for research data, including large and complex data types

- gold Open Access which fosters wider collaboration and increased citations

- maximum visibility for your research: over $100 \mathrm{M}$ website views per year

At BMC, research is always in progress.

Learn more biomedcentral.com/submissions 